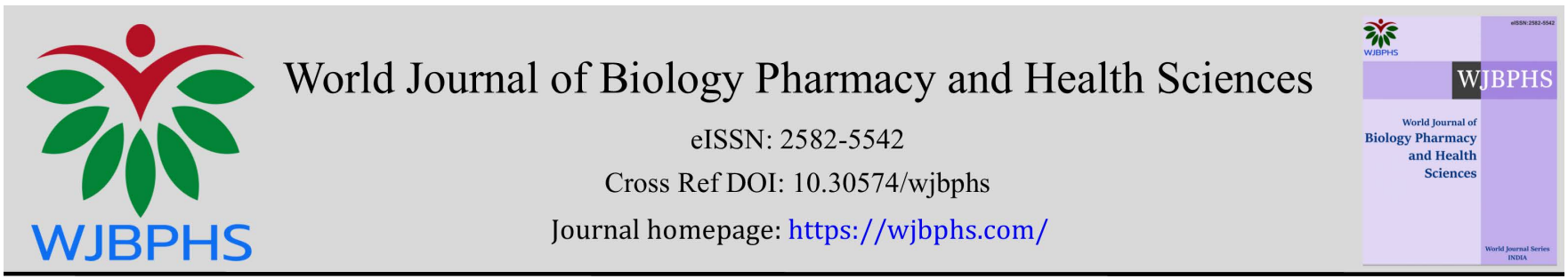

(RESEARCH ARTiCLE)

\title{
Physicochemical and stability profile of castor oil emulsions stabilized using natural and synthetic emulsifiers
}

\author{
Oluwadamilola M Kolawole *, Kayode Q Akinlabi and Boladale O Silva \\ Department of Pharmaceutics and Pharmaceutical Technology, Faculty of Pharmacy, University of Lagos, Lagos, Nigeria.
}

World Journal of Biology Pharmacy and Health Sciences, 2022, 09(02), 060-073

Publication history: Received on 12 January 2022; revised on 15 February 2022; accepted on 15 February 2022

Article DOI: https://doi.org/10.30574/wjbphs.2022.9.2.0043

\begin{abstract}
Background: The quality and stability of castor oil emulsions may be influenced by the type and amount of its constituent emulsifiers. To our knowledge, the emulsifying potential of single, double, and triple-emulsifier systems comprising of Tween 20, acacia gum and methyl cellulose have never been compared. The aim of the work was to formulate castor oil emulsions stabilized using natural and/or synthetic emulsifying agents and evaluate their organoleptic, physicochemical, and stability profiles.
\end{abstract}

Methods: Six types of emulsions were prepared using the spontaneous emulsification method, and characterised in terms of their physical properties; centrifugation analysis, and real-time stability profile at $25^{\circ} \mathrm{C}$ and $40{ }^{\circ} \mathrm{C}$ for 90 days.

Results: FT-IR analysis suggested that castor oil was compatible with all the studied emulsifiers. Tween 20 -stabilised formulation (F2) and acacia gum/methyl cellulose-based samples (F5) exhibited unacceptable organoleptic attributes and/or physical properties. The physical properties of the remaining castor oil macroemulsions were satisfactory: $\mathrm{pH}$ (6.2 to 7.0); electrical conductivity $(2253-4557 \mu \mathrm{S} / \mathrm{cm})$; spreadability $(0.7-2.1 \mathrm{~cm})$; oil globule size $(2.73-3.74 \mu \mathrm{m})$, and creaming index (9-18\%). The emulsions exhibited varying degree of instability over time and with elevated temperatures. The most promising emulsion was acacia gum/methyl cellulose/tween 20-stabilised sample F7: pH: 6.9, electrical conductivity: $4557 \mu \mathrm{S} / \mathrm{cm}$, spreadability: $1.37 \mathrm{~cm}$, oil globule size: $3.74 \mu \mathrm{m}$, creaming index: $9 \%$, and satisfactory stability profile at $25^{\circ} \mathrm{C}$ and $40{ }^{\circ} \mathrm{C}$.

Conclusion: Sample F7 may serve as cream base for dermal and transdermal delivery of active pharmaceutical ingredients intended to treat various local skin problems and systemic diseases.

Keywords: Castor oil emulsions; Tween 20; Acacia gum; Methyl cellulose; Physicochemical; Stability studies

\section{Introduction}

Emulsions are colloidal dispersions that consist of at least two immiscible phases (oily and aqueous) in which one of the components (dispersed phase) is dispersed in the other system (continuous phase), and stabilized by emulsifiers/surfactants [1].

Emulsification of the aqueous and oily phases occur via various mechanisms

- Reduction of surface tension within the oil/water system to values less than 10 dyne/cm [2];

\footnotetext{
* Corresponding author: Oluwadamilola M Kolawole

Department of Pharmaceutics and Pharmaceutical Technology, Faculty of Pharmacy, University of Lagos, Lagos, Nigeria. 
- Creation of mono- or multilayer coating film around the dispersed oil droplets, which reduces interfacial tension; increases droplet-droplet repulsion, preventing droplets aggregation, and promoting the colloidal stability of the emulsions [2, 3].

There are various parameters that determine the type of emulsion formed:

- Proportion of oily phase to aqueous phase, with oil-in-water emulsions formulated when the dispersion phase constitutes more than $45 \%$ of the formulation weight and vice-versa;

- The hydrophile-lipophile balance (HLB) of emulsifying agents, with emulsifiers exhibiting HLB values between 9 and 12 generating oil-in-water emulsions while water-in-oil emulsions are formed when the HLB range of the emulsifier is between 3 and 6 [4].

The physical and chemical compatibility between the active ingredient and other emulsion excipients dictate the stability of the emulsions. For example, anionic emulsifiers are not typically used alongside cationic emulsifiers in an emulsion, to prevent coalescence of the dispersed phase droplets, which may render the drug formulation ineffective [2]. Furthermore, light and air sensitivity as well as microbial susceptibility of pharmaceutical emulsions may affect their stability [2]. Thus, emulsions containing photo-labile ingredients are secured in light resistant containers while anti-oxidants and fungistatic preservatives are included in pharmaceutical emulsions to improve their long term stability [2].

Emulsifiers may be natural or synthetic in nature, and they could be made up of either simple or large molecules [4]. Polyoxyethylene sorbitan monolaurate/polysorbate 20/Tween 20 is a non-ionic, synthetic, low-molecular weight surfactant, with an HLB value of 16.7, and molecular weight of $1.23 \mathrm{kDa}$. The surfactant is generated by the condensation of anhydrous sorbitol with approximately 20 moles of ethylene oxide, followed by reaction with lauric acid [5]. It is generally considered as a safe excipient for food and pharmaceutical applications [5]. Tween 20 typically form monomolecular films around the dispersed phase droplets as well as being adsorbed at the oil/water interface, which promotes repulsion between dispersed phase droplets, decrease interfacial tension, and prevent dispersed globule coalescence [2]. It is frequently used in the cosmetic and pharmaceutical industries as an emulsifying agent to formulate stable oil-in-water topical emulsions due to its non-irritant properties [6]. Tweens/Polysorbates are heat sensitive and will darken when exposed to elevated temperatures. In addition, they are incompatible with alkalis, heavy metal, salts, phenols, and tannic acid resulting in the generation of unstable products. Furthermore, tweens may not be used to stabilize emulsions requiring heat for its preparation [6].

Since most low molecular weight surfactants are relatively toxic, irritant, and constitute environmental pollution, natural molecules such as polysaccharides are alternatively used as emulsifiers to stabilize emulsions due to their biocompatibility and eco-friendliness [7]. Moreover, polysaccharides exert superior emulsifying potential relative to low molecular weight surfactants because the former generates multi-molecular emulsifier films around the dispersed phase droplets while monomolecular films of Tween 20 are created around the dispersed oil globules [2].

Acacia gum or Gum Arabic is a natural, high-molecular weight polysaccharide-based emulsifier, with an average molecular weight of $760 \mathrm{kDa}$ that is obtained from the stems and branches of Acacia senegal (L) Willdenow or Acacia seyal, which are abundantly available at Sub-Saharan Africa [8]. The polysaccharide gum has a neutral or slightly acidic $\mathrm{pH}$, with backbone consisting of 1, 3-linked $\beta$-D-galactopyranosyl units. Acacia gum supports the formation of oil-inwater emulsions due to its hydrophilic nature and favourable HLB value (8.0 to 11.9) [2]. Acacia gum exhibit film forming, microencapsulating, thickening, gelling, adhesive, emulsifying, and stabilizing properties [8, 9]. It exerts its emulsifying potential via multi-molecular film formation around oil droplets as well as interfacial adsorption, resulting in emulsion stabilization [10-12].

Methyl cellulose (MC) is a non-irritant, semi-synthetic cellulose derivative, with an average molecular weight of 10 to $220 \mathrm{kDa}$ and HLB value of 10.5. The polymer is insoluble in ethanol, ether and chloroform, but soluble in glacial acetic acid. It swells in water to form a translucent viscous colloidal solution, with neutral pH [13]. Methyl cellulose has been explored as suspending, binder, adhesive, thickening agent, and stabilizer in food, cosmetic and pharmaceutical products [14]. In order to exert its emulsion stabilizing action, methyl cellulose increases the viscosity of the aqueous phase, decreasing flowability, and improving dispersed phase droplet stability against flocculation and creaming [15].

In recent years, there has been a growing interest towards exploring emulsified systems to encapsulate and deliver lipophilic bioactive ingredients for pharmaceutical and biomedical applications [16]. The pharmaceutical benefits of formulating bioactive oils as emulsions include enhancement of kinetic stability; improved solubilization and 
bioavailability of the oils as well as masking of unpleasant taste [17]. Examples of bioactive oils that have been formulated as emulsions include cinnamon leaf oil [18], sesame oil [19], castor oil [20], [21], olive oil [16], soybean oil [7], palm oil [22], chaulmoogra oil [23], clove oil [24], and eugenol [25].

Various researchers have stabilized bioactive oil based emulsions using either single or multiple emulsifier systems. For example, Zhang and co-workers reported that hydroxypropyl methylcellulose (HPMC; $2 \% \mathrm{w} / \mathrm{v}$ ), methylcellulose (MC; $1.5 \% \mathrm{w} / \mathrm{v})$, gelatin (7 \% w/v), poloxamer $407(\mathrm{~F} 127 ; 8 \% \mathrm{w} / \mathrm{v})$, or poloxamer $188(\mathrm{~F} 68 ; 15 \% \mathrm{w} / \mathrm{v})$, were required to formulate stable soybean oil emulsions [7]. MC-stabilized emulsions exhibited phase separation during autoclaving sterilization-stability studies [7], probably because the elevated temperature conditions increases emulsion viscosity without exerting interfacial emulsifying action. In addition, emulsifier macromolecule chains might be degraded, resulting in its precipitation, aggregation and instability of the emulsion [7]. Overall, poloxamer, hydroxyl propyl methyl cellulose, and methyl cellulose based emulsifiers exhibited improved soybean oil emulsifying potential, in comparison to gelatin [7].

Ologunagba and co-workers established that coconut oil-based capsaicin cream stabilized using mixture of $15 \%$ Grewia mollis mucilage (natural polysaccharide gum) and $10 \%$ HPMC (semi-synthetic polysaccharide) exhibited superior cream stability relative to Grewia gum-stabilised or HPMC-stabilised capsaicin creams [26]. This finding may be due to natural polysaccharide Grewia gum forming multimolecular film around the oil globules (dispersed phase) while HPMC increased the viscosity of the emulsion's aqueous dispersion medium, resulting in the improved stability of the capsaicin creams.

Pale yellowish Castor oil is a vegetable oil extracted from the seeds of the Ricinus communis plant (Family: Euphorbiaceae). It exhibits a density and refractive index of $0.96 \mathrm{~g} / \mathrm{mL}$ and 1.476 , respectively at $28^{\circ} \mathrm{C}$ [27], and its phenolic content is responsible for its stability and flavor [28]. Castor oil is frequently used in the food, cosmetic and pharmaceutical industries due to its rich supply of monounsaturated fatty acid (90\%) as well as bioactive compounds such as phospholipids and vitamin E, including tocopherols or tocotrienols [28-29]. Tocopherol possess anti-cancer activity, anti-oxidant, and anti-inflammatory properties [22, 31], and the amount of delta and gamma forms of tocopherol in castor oil are relatively higher than those reported for olive oil, hazelnut oil, and sunflower oil [28-29].

Kusuma and Sambashiva, 2016 investigated the laxative potential of castor oil emulsions, and stabilised the formulations using 1-4 \% of pre-gelatinized potato starch, acacia gum or methyl cellulose [21]. They reported that pregelatinized potato starch (4\%) displayed superior emulsifying potential relative to acacia gum (4\%) and methyl cellulose $(4 \%)$ in terms of castor oil emulsion stability at ambient temperature after three months [21]. However, the possibility of formulating castor oil emulsions using mixtures of emulsifiers, for potential topical application was not investigated. In addition, the stability of the formulations at elevated temperatures was not investigated. Thus the long term stability profile of the emulsion products could not be predicted.

The biological activities of tocopherol present in castor oil renders it suitable for topical drug delivery. To our knowledge, the potential of single, double and triple natural and/or synthetic emulsifier systems to stabilise castor oil emulsions has never been compared. Therefore, the aim of our study was to formulate castor oil emulsions using optimized concentrations of tween 20, acacia gum and methyl cellulose, and evaluate their physical properties and stability profile, for potential topical application.

\section{Material and methods}

\subsection{Materials}

Castor oil (KTC Edibles Ltd, UK), Tween 20 (LinylGuoli Chemical Co., Ltd, China), Acacia gum, Methyl cellulose (Shijiazhuang Jianxin Cellulose Co., Ltd), propylene glycol (Inner Mongolia Pulis Chemical Co., Ltd, China), methyl paraben (Xian Faithful Biotech. Co., Ltd, China), propyl paraben (Arshine Pharmaceutical Co., Ltd, China). Distilled water was used for all experiments.

\subsection{Methods}

2.2.1. Compatibility studies between castor oil and emulsifiers (Tween 20, Acacia gum and Methyl cellulose) using Attenuated Total Reflectance Fourier-Transform Infrared (ATR FT-IR) Spectroscopy

The compatibility between castor oil and the studied emulsifying agents was determined using Agilent Cary 630 ATRFTIR spectrophotometer (Agilent Scientific, UK). The FT-IR spectra were obtained by scanning pure samples of castor oil, 
tween 20, acacia gum, methyl cellulose as well as physical mixture of castor oil and the emulsifiers from 4000 to 650 $\mathrm{cm}^{-1}$, at a resolution of $8 \mathrm{~cm}^{-1}$. Data was processed based on the average of 32 scans per spectrum generated.

\subsection{Formulation of castor oil emulsions}

Seven types of Castor oil emulsions (F1 to F7) were prepared using the spontaneous emulsification technique. Castor oil, acacia gum, methyl cellulose and propylene glycol constitute the oily phase while aqueous phase comprises of tween 20, methyl paraben, and propyl paraben. Propylene glycol served as the permeation enhancer while mixture of methyl paraben and propyl paraben preserved the emulsions. Samples F1, F3, and F5 were formulated by triturating predetermined amounts of acacia gum and/or methyl cellulose with castor oil, followed by addition of propylene glycol (Table 1). Afterwards, preservative containing water is added to the mixture, which was triturated for 3 min to generate the primary emulsion. Then, additional distilled water was added to the primary emulsion in order to make up the predetermined weight of the castor oil emulsions. The final product was further triturated in the mortar and pestle for 2 min. The finished product was secured in cream jars and stored at room temperature. Similar method was used to prepare sample F4, F6 and F7, though tween 20 was additionally incorporated into the oil/emulsifier/propylene glycol mixture while sample F2 was prepared by mixing castor oil with propylene glycol only before addition of tween 20 (Table 1).

Table 1 Composition of castor oil emulsions

\begin{tabular}{|l|c|c|c|c|c|c|c|}
\hline \multicolumn{1}{|c|}{ Ingredients } & F1 & F2 & F3 & F4 & F5 & F6 & F7 \\
\hline Oily phase (\% w/w) & 35 & 35 & 35 & 35 & 35 & 35 & 35 \\
\hline Castor oil & 4 & - & - & 4 & 4 & - & 4 \\
\hline Acacia gum & - & - & 4 & - & 4 & 4 & 4 \\
\hline Methyl cellulose & 3 & 3 & 3 & 3 & 3 & 3 & 3 \\
\hline Propylene glycol & - & 30 & - & 30 & - & 30 & 30 \\
\hline Aqueous phase (\% w/w) & - & & & & & \\
\hline Tween 20 & 0.5 & 0.5 & 0.5 & 0.5 & 0.5 & 0.5 & 0.5 \\
\hline Methyl paraben & 0.25 & 0.25 & 0.25 & 0.25 & 0.25 & 0.25 & 0.25 \\
\hline Propyl paraben & 0.25 & 100 & 100 & 100 & 100 & 100 & 100 \\
\hline Distilled water to & 100
\end{tabular}

Key: F1- Acacia gum; F2-Tween 20; F3 - Methyl cellulose; F4- Tween 20/Acacia gum; F5-Acacia gum/Methyl cellulose; F6- Tween 20/Acacia gum; F7- Tween 20/Acacia gum/Methyl cellulose

\subsection{Characterization of castor oil emulsions}

\subsubsection{Evaluation of Organoleptic attributes}

The organoleptic attributes of the formulations such as colour and odour were assessed by visual and olfactory evaluation. The texture of the emulsions was evaluated by pressing a small amount of the formulation between the thumb and index finger in order to check for the presence of coarse particles while the consistency of the formulations was assessed based on its homogeneity. Furthermore, the castor oil emulsions were applied to the hands and their ease of removal after washing the body part using tap water was evaluated.

\subsection{Evaluation of physical properties}

\subsection{1. $p H$ determination}

The $\mathrm{pH}$ of the formulations was determined at $25^{\circ} \mathrm{C}$ using a BenchTop Jenway $3510 \mathrm{pH}$ meter (Jenway, UK). The $\mathrm{pH}$ determination was carried out after calibration of the $\mathrm{pH}$ meter using standard buffer solutions. 


\subsection{Conductivity determination}

The conductivity of the emulsions was determined at $25^{\circ} \mathrm{C}$ using the Jenway 4510 Conductivity Meter (Jenway, UK). The electrode was calibrated using gas-free distilled water prior to sample analysis.

\subsection{Viscosity determination}

The viscosities of various formulations were determined using the Brookfield Ametek Viscometer DV-2T (Brookfield Engineering Laboratories, Middleboro, Massachusetts, USA) at $25^{\circ} \mathrm{C}$, according to a previously reported method [26]. The equipment was calibrated and auto-zeroed before use. Formulation F2 was not studied due to its instability. Sample F1, F3-F6 were analyzed using the spindle No. 6 while spindle No. 5 was used to evaluate the viscosity of formulation F7. The samples were subjected to various shear stresses $(10,20,40,80,100$, and $120 \mathrm{rpm})$ in order to evaluate their flow properties.

\subsection{Spreadability evaluation}

The spreadable nature of the formulations was evaluated using an earlier reported method with modification [26]. Each sample $(1 \mathrm{~g})$ was placed between two glass slides $(7.5 \mathrm{~cm} \times 2.5 \mathrm{~cm})$ and $20 \mathrm{~g}$-weight was placed on the topmost glass slides, which facilitated the spread of the formulation. The final spread circle diameter was evident when there was no further spread of the formulation while the initial spread circle diameter was determined prior to the application of the 20 g-weight. The spreadability of the creams was assessed by measuring the difference between the initial and final spread circle diameter of the formulations.

\subsection{Globule size determination using microscopy}

A small portion of each emulsion product was smeared on a clean glass slide, stained with a drop of amaranth solution and covered with a slip. Then, the sample was viewed under the BINO CXI compound microscope (Micron Instruments Ltd., USA). Using the low power objective lens at a magnification x 10, the size of ten randomly selected globules (dispersed phase) was determined and the average particle size of the dispersed phase droplets was recorded.

\subsection{Accelerated Stability Studies: Centrifugation analysis}

The various formulations ( $5 \mathrm{~g}$ ) were transferred into centrifuge vials and secured in the Eppendorf Centrifuge 5810 (Sigma-Aldrich, UK), and the equipment was set at a speed of 4,000 rpm for $10 \mathrm{~min}$ over 2 runs. Afterwards, the creams were observed macroscopically for any evidence of liquefaction or phase separation. The creaming index of the samples was calculated as described by Petrovic and co-workers [32].

$$
\text { Creaming index }(\%)=\text { HS/HE x } 100
$$

Where HS is the height of the cream layer which was measured after the centrifugal analysis, and HE is the total height of the emulsion. The smaller the value of creaming index, the more stable is the emulsion.

\subsection{Short term stability studies at $25 \pm 2{ }^{\circ} \mathrm{C}$ and $40 \pm 2{ }^{\circ} \mathrm{C}$}

The physical stability of the castor oil emulsions secured in vials, was studied under ambient conditions $\left(25 \pm 2{ }^{\circ} \mathrm{C}\right)$, and at elevated temperature $\left(40 \pm 2^{\circ} \mathrm{C}\right)$. The samples were evaluated in terms of $\mathrm{pH}$, colour and odour changes as well as signs of phase separation at $0,7,15,30,60$, and 90 days.

\subsection{Statistical analysis}

All experiments were carried out in triplicates and data presented as mean \pm standard deviation. One way-ANOVA was used for statistical analysis, with $\mathrm{p}<0.05$ indicating significant statistical differences between various data sets.

\section{Results}

\subsection{FT-IR data}

The typical absorption bands for pure castor oil are presented in Fig. $1 \mathrm{a}: 723.1 \mathrm{~cm}^{-1}, 2851 \mathrm{~cm}^{-1}, 2922.2 \mathrm{~cm}^{-1}(\mathrm{C}-\mathrm{H} \mathrm{stretch}$ of methyl groups), $1162.9 \mathrm{~cm}^{-1}, 1740.7 \mathrm{~cm}^{-1}$ (C-O-C ester of carbonyl groups), $1457.4 \mathrm{~cm}^{-1}, 3004.2 \mathrm{~cm}^{-1}$ (C=C alkenyl groups), $3410.5 \mathrm{~cm}^{-1}\left(\mathrm{O}-\mathrm{H}\right.$ bend of primary alcohol) while that of tween 20 was detected at $752.9 \mathrm{~cm}^{-1}, 2873.8 \mathrm{~cm}^{-1}(-\mathrm{C}-$ $\mathrm{H}$ alkyl groups), $1237.5 \mathrm{~cm}^{-1}$ (C-O-C), and $3369.5 \mathrm{~cm}^{-1}$ (-OH stretch). The FT-IR spectrum for castor oil/tween $20 \mathrm{mixture}$ 
revealed characteristic absorption bands evident in both pure castor oil and tween 20 (Fig. 1a), indicating that there was no significant interaction between castor oil and tween 20.

Pure acacia gum exhibited absorption peaks at $894.6 \mathrm{~cm}^{-1}, 1412.7 \mathrm{~cm}^{-1}(\mathrm{C}=\mathrm{C}), 1200.2 \mathrm{~cm}^{-1}(\mathrm{C}-0-\mathrm{C}), 1319.5 \mathrm{~cm}^{-1}, 2914.8$ $\mathrm{cm}^{-1}(\mathrm{C}-\mathrm{H})$, and $3265.1 \mathrm{~cm}^{-1}\left(\mathrm{O}-\mathrm{H}\right.$ stretching vibration). Methyl cellulose absorption bands were detected at $1051.1 \mathrm{~cm}^{-}$ ${ }^{1}$ (C-O-C), $1580.4 \mathrm{~cm}^{-1}$ (C=C), $3503.7 \mathrm{~cm}^{-1}(-\mathrm{OH}), 1319.6 \mathrm{~cm}^{-1}, 2885.0 \mathrm{~cm}^{-1}$, and $2907.3 \mathrm{~cm}^{-1}$ (C-H). The functional groups evident in the spectra for pure castor oil and acacia gum were replicated in the spectrum of their mixture (Fig. 1b). A similar trend was observed with the spectra of pure castor oil and methyl cellulose in comparison to castor oil/methyl cellulose mixture (Fig. 1c).

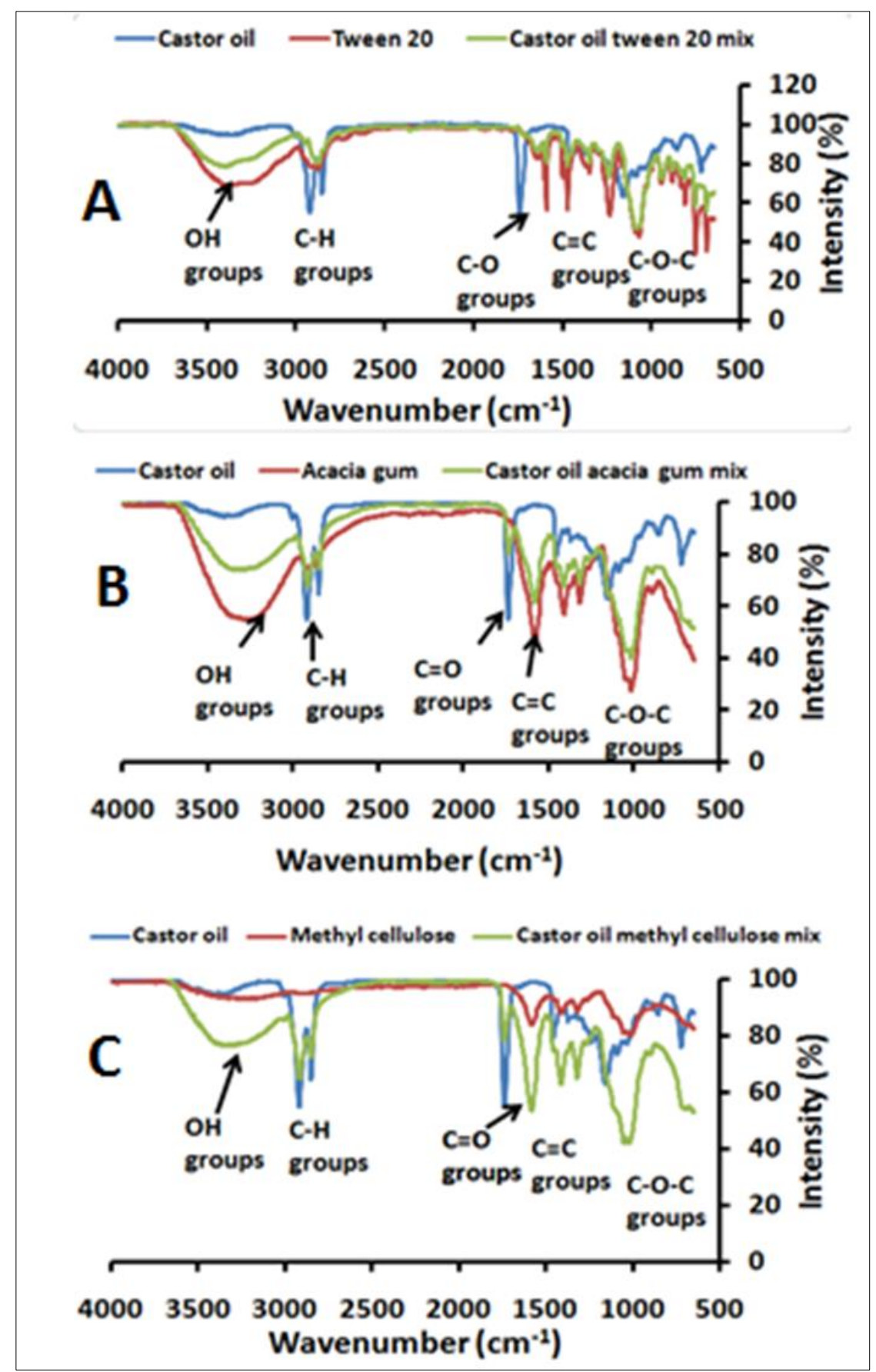

Figure 1 FT-IR spectra of (a) castor oil, tween 20 and castor oil/tween 20 mixture; (b) castor oil, acacia gum and castor oil/acacia gum mixture; (c) castor oil, methyl cellulose and castor oil/methyl cellulose mixture, confirming compatibility between castor oil and the studied emulsifiers 
The FT-IR spectrum of the physical mixture of castor oil, tween 20, acacia gum and methyl cellulose (Fig. 2) revealed absorption peaks at $752.9 \mathrm{~cm}^{-1}$ (-CH group) and $1237.5 \mathrm{~cm}^{-1}$ (C-O-C group) from tween 20; moderate shift in peak position from 894.6 to $887.1 \mathrm{~cm}^{-1}$ depicting $\mathrm{C}-\mathrm{H}$ groups from acacia gum as well as an absorption peak at $1021.2 \mathrm{~cm}^{-1}$ representing $\mathrm{C}-\mathrm{O}-\mathrm{C}$ groups in acacia gum; $\mathrm{C}=\mathrm{CH}$ groups evident at $1412.7 \mathrm{~cm}^{-1}$ in pure acacia gum shifted moderately to $1408.9 \mathrm{~cm}^{-1}$ in the physical mixture. Also, there was a moderate shift in the absorption peak for ether groups (C-O-C) in castor oil from $1162 \mathrm{~cm}^{-1}$ (pure sample) to $1058 \mathrm{~cm}^{-1}$ (physical mixture, CTAM). Furthermore, there were various moderate shifts in the absorption peaks of $\mathrm{C}=\mathrm{C}$ groups of methyl cellulose: pure sample $\left(1580.4 \mathrm{~cm}^{-1} \mathrm{and}_{2885} \mathrm{~cm}^{-1}\right)$ versus physical mixture of castor oil and the three emulsifiers $\left(1591.6 \mathrm{~cm}^{-1}\right.$ and $\left.2870 \mathrm{~cm}^{-1}\right)$.

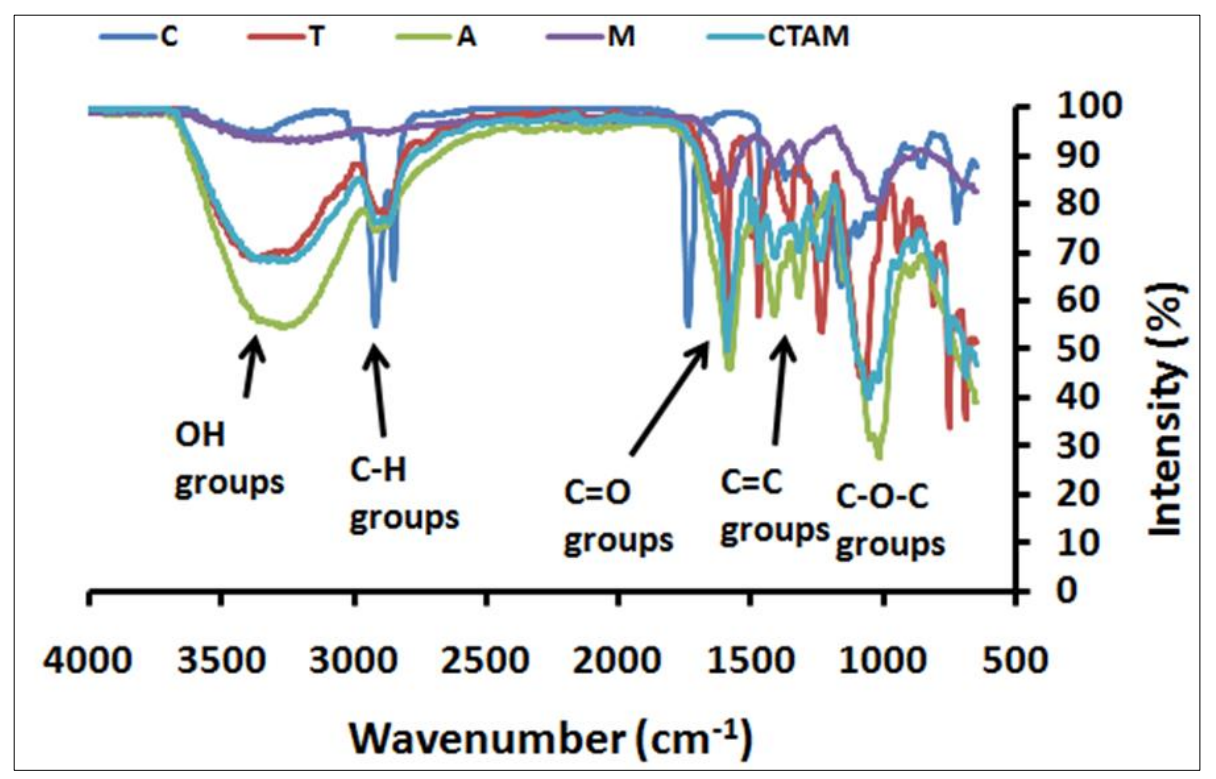

Figure 2 FT-IR spectra of castor oil, tween 20, acacia gum, methyl cellulose and castor oil/tween 20/acacia gum/methyl cellulose mixture

\subsection{Organoleptic attributes and Physical properties}

Tween 20-containing formulations (F2, F4, F6, and F7) were whitish while acacia gum and/or methyl cellulosestabilized samples (F1, F3, F5) were off-white in colour. Apart from samples F2 and F5, all other formulations were odourless, homogenous, with smooth texture. Sample F2 exhibited creaming effect and limited emulsion stability while formulation F5 was very viscous. After applying all the seven samples to the hand, they were readily removed by washing the body part with running water.

Table 2 Physical properties of the castor oil-in-water emulsions

\begin{tabular}{|c|c|c|c|c|c|}
\hline $\begin{array}{c}\text { Emulsion } \\
\text { code }\end{array}$ & $\mathbf{p H}$ & $\begin{array}{c}\text { Electrical } \\
\text { Conductivity } \\
(\boldsymbol{\mu S} / \mathbf{c m})\end{array}$ & $\begin{array}{c}\text { Average } \\
\text { Viscosity }(\mathbf{c P}) \\
\text { at 40 rpm }\end{array}$ & $\begin{array}{c}\text { Spreadability } \\
\mathbf{( c m )}\end{array}$ & $\begin{array}{c}\text { Globule } \\
\text { size }(\boldsymbol{\mu m})\end{array}$ \\
\hline F1 & $7.0 \pm 0.1$ & $1609 \pm 2$ & 7950 & $0.83 \pm 0.06$ & $2.93 \pm 0.57$ \\
\hline F2 & $4.2 \pm 0.1$ & $1115 \pm 3$ & $\mathrm{ND}$ & $2.50 \pm 0.10$ & $3.23 \pm 1.49$ \\
\hline F3 & $6.5 \pm 0.1$ & $2253 \pm 15$ & 11480 & $0.73 \pm 0.15$ & $3.74 \pm 0.83$ \\
\hline F4 & $6.2 \pm 0.1$ & $4300 \pm 10$ & 5625 & $2.10 \pm 0.10$ & $3.43 \pm 1.09$ \\
\hline F5 & $6.8 \pm 0.1$ & $4540 \pm 20$ & 23450 & $0.70 \pm 0.10$ & $3.54 \pm 1.28$ \\
\hline F6 & $6.4 \pm 0.1$ & $4473 \pm 12$ & 4200 & $1.00 \pm 0.10$ & $2.73 \pm 0.96$ \\
\hline F7 & $6.9 \pm 0.1$ & $4557 \pm 6$ & 8910 & $1.37 \pm 0.06$ & $3.74 \pm 0.96$ \\
\hline
\end{tabular}

Key: ND = not determined; F1 = $4 \%$ acacia gum; F2 $=30 \%$ tween $20 ; \mathrm{F} 3=4 \%$ methyl cellulose; F4= $4 \%$ acacia gum/30\% Tween $20 ; \mathrm{F} 5=4 \%$ acacia gum $/ 4 \%$ methyl cellulose; F6 $=4 \%$ methyl cellulose $/ 30 \%$ tween $20 ; \mathrm{F} 7=4 \%$ acacia gum $/ 4 \%$ methyl cellulose/ $30 \%$ tween 20 
Table 2 depicts some physical properties of the castor oil-in-water emulsions. Most of the formulations exhibited mildly acidic to neutral $\mathrm{pH}(6.2-7.0)$ except sample $\mathrm{F} 2$ with a $\mathrm{pH}$ of $4.2 \pm 0.1$. The electrical conductivity values of the stable formulations ranged from 1609 to $4557 \mu \mathrm{S} / \mathrm{cm}$. Tween 20-based sample F2 displayed the lowest electrical conductivity $(1115 \pm 3 \mu \mathrm{S} / \mathrm{cm})$ while the greatest electrical conductivity was exhibited by sample F7 stabilised using tween 20 , acacia gum and methyl cellulose ( $4557 \pm 6 \mu \mathrm{S} / \mathrm{cm})$.

The viscosity of F2 was not determined due to its poor colloidal instability. The average viscosities of the studied formulations at a shear rate of 40 rpm were: F6 (4200 cP) < F4 (5625 cP) < F1 (7950 cP) < F7 (8910 cP) < F3 $(11480$ $\mathrm{cP})<\mathrm{F} 5(23450 \mathrm{cP})$ (Table 2). The viscosities of the studied emulsions decreased as shear rates was increased from 10 rpm to $120 \mathrm{rpm}$ (Figure 3).

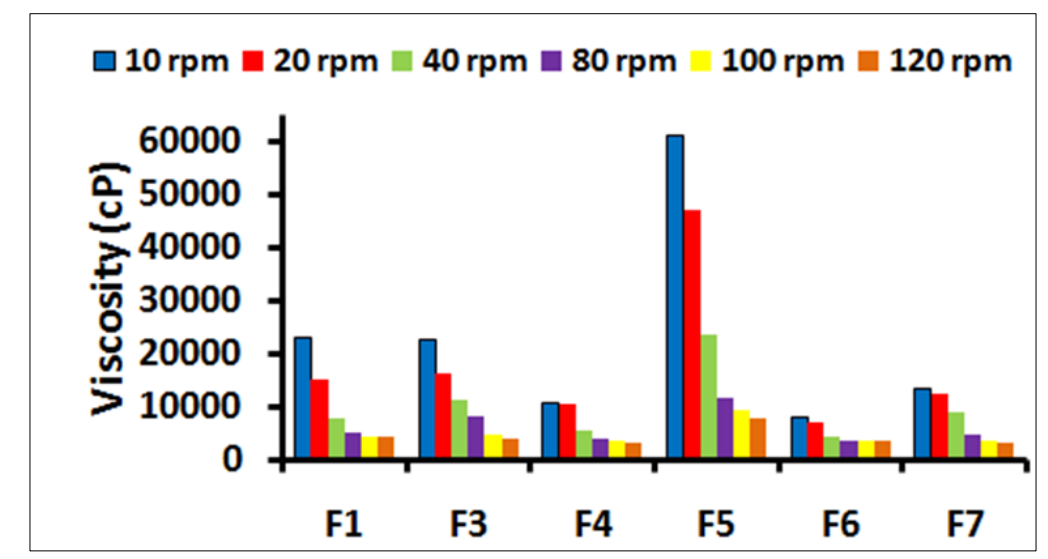

Figure 3 Average viscosity values of castor oil emulsions at $25 \pm 1^{\circ} \mathrm{C}$, with increasing shear rate (10 rpm to $120 \mathrm{rpm}$ )

The castor oil emulsions are presented in order of increasing spreadability: F5 $(0.70 \pm 0.10 \mathrm{~cm})<\mathrm{F} 3(0.73 \pm 0.15 \mathrm{~cm})<$ $\mathrm{F} 1(0.83 \pm 0.06 \mathrm{~cm})<\mathrm{F} 6(1.00 \pm 0.10 \mathrm{~cm})<\mathrm{F} 7(1.37 \pm 0.06 \mathrm{~cm})<\mathrm{F} 4(2.10 \pm 0.10 \mathrm{~cm})<\mathrm{F} 2(2.50 \pm 0.10 \mathrm{~cm})($ Table 2$)$.

The globule sizes of the conventional castor oil emulsions ranged from 2.73 to $3.74 \mu \mathrm{m}$. Formulation F3 stabilised using methyl cellulose exhibited globule size of $3.74 \mu \mathrm{m}$. Incorporation of acacia gum into the emulsifier system reduced the globule size of the formulation F5 to $2.73 \pm 0.96 \mu \mathrm{m}$. Furthermore, inclusion of tween 20 as the third emulsifier resulted in an increase of oil droplet size to $3.74 \mu \mathrm{m}$ (F7) (Table 2).

\subsection{Accelerated and Real-time Stability data}

\subsubsection{Accelerated Stability data: Centrifugation}

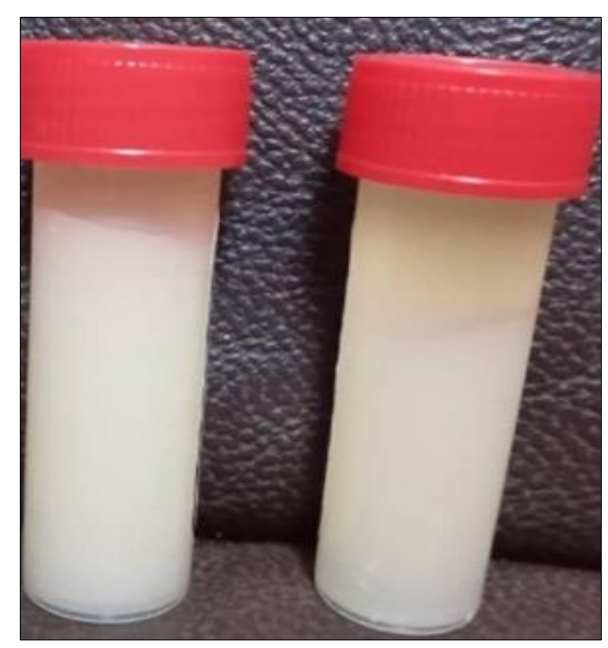

Figure 4 Samples F5 and F1 showing varying degree of phase separation after centrifugation at $4000 \mathrm{rpm}$ for $10 \mathrm{~min}$ over two cycles 
Sample F1 stabilised using acacia gum and sample F5 stabilised using mixture of acacia gum and methyl cellulose have been provided as representative samples to depict varying degree of phase separation exhibited by castor oil emulsions after centrifugation at $4000 \mathrm{rpm}$ for $10 \mathrm{~min}$ over two cycles (Fig. 4).

The calculated extent of creaming for the investigated castor oil emulsions is presented in Table 3 . The formulations presented in order of decreasing creaming rate and increasing stability: F2 (93\%) < F3, F4 (18 \%) < F1 (16 \%) < F6 (11 $\%)<$ F5, F7 (9\%).

Table 3 Accelerated Stability profile of castor oil emulsions: Centrifugation at $4000 \mathrm{rpm}, 10 \mathrm{~min}, 2$ cycles

\begin{tabular}{|c|l|c|c|}
\hline Formulation code & Emulsifier & Degree of phase separation (cm) & Creaming index (\%) \\
\hline F1 & Acacia gum & 0.7 & 16 \\
\hline F2 & Tween 20 & 4.2 & 93 \\
\hline F3 & Methyl cellulose & 0.8 & 18 \\
\hline F4 & Acacia gum/Tween 20 & 0.8 & 18 \\
\hline F5 & Acacia gum/Methyl cellulose & 0.4 & 11 \\
\hline F6 & $\begin{array}{l}\text { Methyl cellulose/ } \\
\text { Tween 20 }\end{array}$ & 0.5 & 9 \\
\hline F7 & $\begin{array}{l}\text { Acacia gum/Methyl cellulose/ } \\
\text { Tween 20 }\end{array}$ & 0.4 & \\
\hline
\end{tabular}

\subsection{Real-time Stability data at $25 \pm 2$ ? and $40 \pm 2$ ?}

The studied samples were physically stable immediately after preparation of the emulsions but formulation F2 exhibited short-lived stability in the form of emulsion creaming and oil droplet coalescence over time. There was no remarkable change in the $\mathrm{pH}$, colour and odour of the studied formulations after storage at ambient temperature and elevated temperatures but they displayed varying degree of phase separation over the 90-day study period (Table 4). The stability profile of the castor oil emulsions were studied at $25{ }^{\circ} \mathrm{C}$ and $40{ }^{\circ} \mathrm{C}$ and they are presented in order of increasing stability: F2 <F1 <F3 <F4 < F5 < F6 < F7.

Table 4 Stability profile of studied formulations stored at $25 \pm 2{ }^{\circ} \mathrm{C}$ and $25 \pm 2{ }^{\circ} \mathrm{C}$ for 90 days

\begin{tabular}{|l|c|c|c|c|c|c|c|c|c|c|c|c|c|c|}
\hline \multirow{2}{*}{ Days } & \multicolumn{4}{|l|}{ Storage temperature: $25 \pm$ 2 } & \multicolumn{4}{|c|}{ Storage temperature: $40 \pm 2$ ? } \\
\cline { 2 - 16 } & F1 & F2 & F3 & F4 & F5 & F6 & F7 & F1 & F2 & F3 & F4 & F5 & F6 & F7 \\
\hline 0 & -- & ++ & -- & -- & -- & -- & -- & -- & ++ & -- & -- & -- & -- & -- \\
\hline 7 & -- & ++ & -- & -- & -- & -- & -- & -- & ++ & -- & -- & -- & -- & -- \\
\hline 15 & -- & ++ & -- & -- & -- & -- & -- & -+ & ++ & -- & -- & -- & -- & -- \\
\hline 30 & -+ & ++ & -+ & -- & -- & -- & -- & -+ & ++ & -+ & -+ & -- & -- & -- \\
\hline 60 & -+ & ++ & -+ & -+ & -+ & -+ & -- & -+ & ++ & -+ & -+ & ++ & -+ & -- \\
\hline 90 & ++ & ++ & -+ & ++ & ++ & -+ & -- & ++ & ++ & -+ & -+ & ++ & -+ & -+ \\
\hline
\end{tabular}

Key: -- = absence of phase separation after predetermined time period; $-+=$ partial phase separation after predetermined time period; $++=$ total phase separation after predetermined time period; F1 = $4 \%$ acacia gum; F2 $=30 \%$ tween $20 ; \mathrm{F} 3=4 \%$ methyl cellulose; F4= $4 \%$ acacia gum $/ 30 \%$ Tween 20; F5 = $4 \%$ acacia gum $/ 4 \%$ methyl cellulose; F6 = $4 \%$ methyl cellulose $/ 30 \%$ tween $20 ; \mathrm{F} 7=4 \%$ acacia gum/4 $\%$ methyl cellulose/ $30 \%$ tween 20

\section{Discussion}

The emulsifying potential of tween 20 (synthetic low molecular weight surfactant), acacia gum (natural polysaccharide) and methyl cellulose (semi-synthetic polysaccharide) has been investigated to formulate pharmaceutically acceptable castor oil-in-water emulsions. The formulations were stabilised using single or multiple emulsifier system(s). The 
emulsifier mixture was carefully chosen because structural differences between emulsifiers could transform a highly stable emulsion into an unstable formulation [2].

The hydrophile-lipophile balance (HLB) values of tween 20, acacia gum, and methyl cellulose are 16.7, 8.0-11.9, and 10.5, respectively [33]. The studied emulsifiers exhibited an average HLB value of 13, which was comparable to the required HLB value (14) for the preparation of stable castor oil emulsions.

FT-IR spectroscopy is an established technique to study interactions between bioactive agents and excipients, with shifts in the FT-IR absorption bands as well as appearance and disappearance of absorption peaks in the physical mixture of materials, indicating interactions amongst constituent functional groups [32, 35]. The obtained FT-IR spectra for castor oil, acacia gum, methyl cellulose, and tween 20 were similar to those reported in literature [36-39].

Distinctive absorption peaks were evident in the FT-IR spectra of pure castor oil, tween 20, acacia gum and methyl cellulose were also observed in their physical mixture, with moderate shift in the absorption region, revealing that castor oil was compatible with all the studied emulsifiers. All the investigated emulsifiers are non-ionic in nature, thus they may be used to formulate non-irritant topical emulsions. Furthermore, there will be reduced tendency for the emulsion products to become chemically unstable because the emulsifiers are chemically compatible.

During preformulation studies, various castor oil emulsions were stabilized using 1-4 \% of acacia gum; 1-4 \% of methyl cellulose, and 5-30\% of tween 20, and the most promising castor oil emulsions were stabilised using single and combination of emulsifier system(s): acacia gum (4\%), methyl cellulose (4\%), and tween 20 (30\%). The relatively high concentrations of emulsifiers used to stabilise castor oil emulsions facilitated the adsorption of greater amount of emulsifier molecules onto the oil droplet surfaces, improving their resistance against creaming and coalescence [7].

Despite stabilizing castor oil emulsions using $30 \%$ of tween 20, the castor oil emulsion remained unstable, probably due to the monomolecular film of tween 20 generated around castor oil droplets, which may be prone to collapse, increasing droplet-droplet attraction, and promoting oil globule flocculation, creaming and coalescence [2]. This finding is in good agreement with earlier studies by Sapei and coworkers that revealed that the emulsifying effect of tween 20 is typically short-lived because the emulsifier leaches out of the oil-water interface, reducing the stability of palm oil emulsions [22].

In addition, sample F5 emulsified using acacia gum and methyl cellulose was not appealing aesthetically because they were highly viscous, which may be due to the synergistic effect between acacia gum and methyl cellulose, with multimolecular acacia gum films generated around castor oil droplets while methyl cellulose improved the viscosity of the aqueous continuous phase [2], which may compromise the spreadability and extrudability of the emulsion from packaging tubes.

On the other hand, the remaining formulations (F1, F3, F4, F6 and F7) exhibited appealing organoleptic attributes in terms of acceptable colour and odour, smooth texture, homogenous, and no signs of phase separation. This finding could be attributed to the inclusion of polymeric emulsifiers, which either form multimolecular emulsifier films around the castor oil droplets and/or improve the viscosity of the aqueous dispersion medium, stabilizing the emulsion [4,32, 40].The incorporation of tween 20 into acacia gum and methyl cellulose-stabilised formulation transformed an aesthetically unacceptable emulsion (F5) to a formulation with satisfactory organoleptic attributes, physical properties, and stability profile (F7).

Castor oil emulsions intended for topical application should be non-irritant to the skin, with pH ranges of 5.5 to 7.0 [40]. Furthermore, the chemical stability and effectiveness of topical formulations is dictated by their pH profiles, as highly acidic formulations such as sample F2 (pH 4.2) may be prone to physical instability and therapeutic inactivity. Conversely, castor oil emulsions stabilized using various combinations of tween 20, acacia gum, and methyl cellulose were physically stable and exhibited satisfactory pH (6.2 to 7.0), suggesting that they have low skin sensitization index.

The electrical conductivity of castor oil emulsions may be dependent on the emulsifying conditions, including the nature of emulsifiers used to stabilise the emulsions. Moreover, highly stable oil-in-water emulsions exhibits high conductivity values [41]. Tween 20 stabilised emulsion F2 exhibited the least physical stability and electrical conductivity value of $1115 \pm 2 \mu \mathrm{S} / \mathrm{cm}$, suggesting that low molecular weight surfactants may not be suitable to emulsify fixed vegetable oil such as castor oil. Formulation F3 stabilised using methyl cellulose exhibited superior conductivity relative to acacia gum-stabilised systems (F1) (1609 $\pm 2 \mu \mathrm{S} / \mathrm{cm}$ versus $2253 \pm 15 \mu \mathrm{S} / \mathrm{cm})$, probably due to the semi-synthetic nature of methyl cellulose which makes it less prone to microbial degradation and instability than acacia gum, which is a natural polysaccharide. 
According to Stoke's equation, the velocity of creaming is indirectly proportional to the viscosity of the dispersion medium [42]. Emulsifiers that increase the viscosity of the continuous medium could improve the overall stability of the emulsions [43]. Formulation F4-F7 stabilised using two to three types of emulsifiers displayed greater electrical conductivity values (4300-4557 $\mu \mathrm{S} / \mathrm{cm})$ than those stabilised using a single emulsifier $(1115-2253 \mu \mathrm{S} / \mathrm{cm})$. This may due to the emulsifiers increasing the viscosity of the dispersion medium as well as decreasing oil globule size. This finding is in good agreement with studies by Ologunagba and co-workers, where they reported that capsaicin creams stabilised using two varieties of polymeric emulsifiers ( $5 \%$ polysaccharide Grewia gum and $10 \%$ hydroxypropyl methyl cellulose) exhibited greater conductivity values $(1812 \pm 18 \mu \mathrm{S} / \mathrm{cm})$ than those stabilised using only $10 \% \mathrm{HPMC}(1127$ $\pm 13 \mu \mathrm{S} / \mathrm{cm})[26]$.

The shear-thinning behaviour of castor oil emulsions may be due to the alignment of the castor oil droplets and emulsifier polymeric chains in the direction of the shearing forces, thereby reducing viscosity of the formulations [16]. Also, there were significant statistical differences between the viscosities of the formulations when the shear stress applied to the castor oil products was doubled $(\mathrm{p}<0.05)$. These findings revealed that the formulations may be extrudable when shear stress is applied to it through pumping of the emulsion product from packaging tubes. The inclusion of tween 20 into the acacia gum and/or methyl cellulose emulsifier system used to stabilize castor oil emulsions resulted in the overall reduction in the viscosity of the castor oil formulations (Table 2), probably because tween 20 leaches out of the oil-water interface into the aqueous continuous phase [22], decreasing the overall viscosity of the emulsions.

Sample F1, F3, F4, F6 and F7 exhibited satisfactory spreadability $(0.83-2.10 \mathrm{~cm})$, indicating that they could be readily applied, rubbed in, and retained on the skin surface. The relatively low spreadability values recorded for the studied samples may be due to the small weight used for the experiment $(20 \mathrm{~g})$. The spreadable nature of $\mathrm{F} 2(2.50 \pm 0.10 \mathrm{~cm})$ may be und esirable for topical application as the formulation may run-off the skin surface, limiting the duration of action of the loaded bioactive agent, and resulting in therapeutic failure. In addition, formulation F5 may not be readily spread over the skin surface $(0.70 \pm 0.10 \mathrm{~cm})$. Formulation F4 stabilised using acacia gum and tween 20 as well as acacia gum/methyl cellulose/tween 20-stabilised sample F7 exhibited satisfactory spreadability of $2.10 \mathrm{~cm}$ and $1.37 \mathrm{~cm}$ respectively, suggesting that the cosmetic appeal of castor oil emulsions may be improved by stabilizing the formulation using mixture of tween 20 and polysaccharide emulsifiers such as methyl cellulose and/or acacia gum.

The oil droplet size of an oil-in-water emulsion is dependent on the type and concentration of the oily phase, as well as the homogenization method [44]. The studied formulations were prepared using mortar and pestle, resulting in the formation of conventional emulsions with mean oil droplet diameter greater than $200 \mathrm{~nm}$. Despite the fact that formulation F7 prepared using the three types of emulsifiers exhibited oil droplet size of $3.74 \pm 0.96 \mu \mathrm{m}$, it still exhibited the greatest degree of physical stability. Conversely, tween 20-stabilised sample F2, with relatively small oil droplet diameter was the least stable formulation, probably due to its broad oil droplet distribution $(3.23 \pm 1.49 \mu \mathrm{m})$. These findings revealed that the average dispersed phase droplet size as well as dispersed phase globule size distribution is an important determinant for the stability of castor oil emulsions.

Kusuma and Sambashiva reported that the oil globule size of conventional castor oil emulsions containing $32 \%$ of castor oil and stabilized using $4 \%$ acacia gum and $4 \%$ methyl cellulose was $2.1 \mu \mathrm{m}$ and $2.0 \mu \mathrm{m}$, respectively [21]. The current study revealed that castor oil emulsions containing $35 \%$ of castor oil and stabilised using similar amount of acacia gum and methyl cellulose were 2.9 and $3.7 \mu \mathrm{m}$, respectively. These disparities in oil droplet size may be associated with differences in the concentration of the castor oil used to formulate the castor oil emulsions. Also, the present study incorporated excipients used to formulate dermal and transdermal dosage forms such as propylene glycol, methyl paraben and propyl paraben, which were not used in the earlier reported study [21].

There was good correlation between the accelerated and real time stability data obtained for the castor oil emulsions studied over 90 days at $25 \pm 2{ }^{\circ} \mathrm{C}$. Acacia gum based samples F1, F3 and F5 exhibited temperature and time-dependent reduction in their physical stability because natural polysaccharidic emulsifiers are prone to microbial degradation [2]. On the other hand, Acacia gum/ methyl cellulose/ tween 20 stabilised sample F7 displayed improved resistance to phase separation in comparison to other studied formulations during the accelerated and real-time stability studies, suggesting that the three types of emulsifiers may be required to formulate castor oil emulsions hat would remain stable for prolonged period of time. In addition, these castor oil based products would be resistant to packaging, storage and environmental stresses [26]. 


\section{Conclusion}

Castor oil emulsions stabilized using single, double and triple emulsifier systems consisting of tween 20, acacia gum and methyl cellulose were evaluated in terms of their physical properties and stability profile. Physicochemical and stability results clearly showed that tween 20 (30\%) alone could not stabilize castor oil emulsions. Overall, sample F7 stabilized using acacia gum, methyl cellulose, and tween 20 was the most promising emulsion in terms of satisfactory physical properties and stability profile. Thus, sample F7 could be used as a cream base to be incorporated with active pharmaceutical ingredients, for the preparation of dermal and transdermal dosage forms.

\section{Compliance with ethical standards}

\section{Acknowledgments}

The authors are grateful to Dr. M. O. Ilomuanya of the Department of Pharmaceutics and Pharmaceutical Technology; Mr. P. D. Ojobor, of the Central Research Laboratory, College of Medicine; Mr. M. Olajide of the Pharmaceutical Chemistry Department; Mr. U. AbdulRahman and Mr. H.E. Anibena of the Department of Pharmaceutical Microbiology and Biotechnology, University of Lagos, Lagos, Nigeria for their support during the research project.

\section{Disclosure of conflict of interest}

The authors declare that there is no conflict of interest.

\section{Authors' contributions}

OMK conceived; OMK and BOS designed the study; KQA and OMK carried out the experimental studies while OMK drafted the manuscript; OMK and BOS revised the manuscript. All authors have read and approved the final manuscript.

\section{References}

[1] Maphosa Y, Jideani V. Factors affecting the stability of emulsions stabilized by biopolymers. In: Science and Technology Behind Nanoemulsions (Ed. Karakus S). $2018 . \quad$ Available from: https://www.intechopen.com/chapters/60140. [cited 2021 Dec 20].

[2] Madaan V, Chanana A, Kataria M, Bilandi A. Emulsion Technology and Recent Trends in Emulsion Applications. Int Res J Pharm. 2014; 5(7): 533-542.

[3] Tamilvanan ST, Kumar B, Senthilkumar S, Baskar R. Stability Assessment of Injectable Castor oil-based nanosized emulsion containing cationic droplets stabilized by poloxamer-chitosan emulsifier films. AAPS PharmSciTech. 2010; 11(2): 904-909.

[4] Kulkarni VS, Shaw C. Surfactants, lipids and surface chemistry. In: Essential Chemistry for Formulators of Semisolid and Liquid Dosages. 2016; 5-19.

[5] PubChem. Compound Summary: Polysorbate $20 . \quad$ Available from: https://pubchem.ncbi.nlm.nih.gov/compound/Polysorbate-20. [cited 2021 Oct 11).

[6] Reynolds J. Martindale: The Extra Pharmacopoeia, 30th ed., London: Pharmaceutical Press. 1993.

[7] Zhang M, Yang B, Liu W, Li S. Influence of hydroxypropyl methylcellulose, methylcellulose, gelatin, poloxamer 407 and Poloxamer 188 on the formation and stability of soybean oil-in-water emulsions. Asian J Pharm Sci. 2017; 12: 521-531.

[8] Masuelli MA. Hydrodynamic properties of Whole Arabic Gum. Amer J Food Sci Technol. 2013; 1(3): 60-66.

[9] El-Kheir MK, Yagoub AE, Baker AA. Emulsion-stabilizing effect of Gum from Acacia Senegal (L) Willd: The role of quality and grade of gum, oil type, temperature, stirring time and concentration. Pakistan J Nutr. 2008; 7(3): 395399.

[10] Farzi M, Emam-Djomeh Z, Mohammadifar M. A comparative study on the emulsifying properties of various species of gum tragacanth. Int J Biol Macromol. 2013; 57: 76-82.

[11] Garcia MC, Alfaro MC, Calero N, Munoz J. Influence of polysaccharides on the rheology and stabilization of $\alpha$ pinene emulsions. Carbohydr Polym. 2014; 105: 177-183. 
[12] Xu D, Zhang J, Cao Y, Wang J, Xia J. Influence of microcrystalline cellulose on the microrheological property and freeze-thaw stability of soybean protein hydrolysate stabilized curcumin emulsion. LWT-Food Sci. Technol. 2016; 66: 590-597.

[13] ACS. Chemical Abstract Service Database List: Methyl cellulose. Chemical Book. 2021. Available from: http//www.chemicalbook.com/chemicalproductproperty_EN_CB3474718 [Cited 2021 Sep. 08].

[14] Brady J, Durig T, Lee P, Li J. Polymer properties and characterization. In: Developing Solid Oral Dosage Forms: Pharmaceutical Theory and Practice, Qiu Y, Chen Y, Zhang G, Yu L, Mantri R., (Eds)., 2nd ed., Pharmaceutical Press. 2017; 1127-1160.

[15] Futamura T, Kawaguchi M. Characterization of paraffin oil emulsions stabilized by hydroxypropyl methyl cellulose. J. Colloid Inter Sci. 2012; 367: 55-60.

[16] Aranbicia C, Navarro-Lisboa R, Zuniga RN. Application of CMC as a thickener on Nanoemulsions based on olive oil: Physical Properties and Stability. J Polym Sci. 2016; 2016: 1-10.

[17] Jemaa M, Falleh H, Ksouri R. Encapsulation of Natural Bioactive Compounds: Nanoemulsion formulation to enhance essentials activities. In: Microencapsulation - Processes, Technologies and Industrial applications, Salaun F (Ed.). 2019; 1-15.

[18] Zainol NA, Ming TS, Darwis Y. Development and characterization of cinnamon leaf oil nanocream for topical application. Indian J Pharm Sci. 2015; 77: 422-433.

[19] Kowalska M, Zbikowska A. Study of Stability of sesame oil-in-water emulsions determined using an optical analyzer and measurement of particle size and distribution. J Dispers. Sci Technol. 2016; 37 (10): 1408-1414.

[20] Zhang D, Lin Y, Li A, Tarasov V. Emulsification for castor biomass oil. Front. Chem. Sci. Eng. 2011; 5(1): 96-101.

[21] Kusuma R, Sambashiva R. Design, Development and Evaluation of Castor oil Emulsion by using Ipomoea Batata Starch as o/w Emulsifier. Int J Med. Pharm. Sci. 2016; 2 (1): 115-118.

[22] Sapei L, Sandy I, Suputra I. The effect of different concentrations of tween 20 combined with rice husk silica on the stability of o/w emulsion: A kinetic study. Mater. Sci Eng. 2017; 273: 1-7.

[23] Panonnummal R, Shammika P, Aiswanya S, Anjaneyan G, Jayakumar R, Sabitha M. Chaulmoogra oil based methotrexate loaded topical nanoemulsion for the treatment of psoriasis. J Drug Deliv Sci Tech. 2019; 49: 463476.

[24] Shahavi M, Hosseini M, Ighanshahi M, Meyer R, Darzi G. Evaluation of critical parameters for preparation of stable clove oil nanoemulsion. Arab J Chem. 2019; 12(8): 3225-3230.

[25] Yang Q, Sui Z, Lu W, Corke H. Soybean lecithin-stabilized oil-in-water emulsions increase the stability and in vitro bioaccessibility of bioactive nutrients. Food Chem 2021; 338: 128071.

[26] Ologunagba MO, Kolawole OM, Echerenwa AN, Silva B. Development and Characterization of capsaicin creams formulated with Grewia mucilage-HPMC base. J Sci Pract. Pharm. 2020; 7(1): 365-375.

[27] Yeboah A, Ying S, Lu J, Xie Y, Amoanimaa-Dede H, Boateng KGA, Chen M, Yin X. Castor oil (Ricinus communis): a review on the chemical composition and physicochemical properties. Food Sci Technol. 2020; 1-15.

[28] Sedeek S, El-Ghobashy R, Tawfik M. Thermal stability of cottonseed oil mixed with jojoba or castor oil during frying process. J Biol. Chem. Environ. Sci. 2012; 7(2): 39-56.

[29] Said G, Daniel P, Badr K, Mohamed I, Charrouf Z. Chemical Characterization and oxidative stability of castor oil grown in Morocco. J Chem. 2016; 4(2): 279-284.

[30] Sbihi HM, Nehdi IA, Mokbli S, Romdhani-Younes M, Al-Resayes . Hexane and ethanol extracted seed oils and leaf essential compositions from two castor oil plant (Ricinus communis L) varieties. Ind. Crops Prod. 2018; 122: 174-181.

[31] Edem DO. Palm oil: biochemical, physiological, nutritional, hematological and toxicological aspects: A Review. Plant Foods Hum. Nutr. 2002; 57 (3-4): 319-341.

[32] Petrovic LB, Sovilj VJ, Katona JM, Milanovic JL. Influence of polymer-surfactant interactions on o/w emulsion properties and microcapsule formation. J. Colloid Interface Sci. 2010; 342 (2): 333-339.

[33] Sinko PJ, Singh Y. Martin's Physical Pharmacy and Pharmaceutical Sciences: The design and manufacture of medicines, 6th ed., Lippincott Williams \& Wilkins. 2010. 
[34] Ilomuanya MO, Elesho RF, Amenaghawon AN, Adetuyi AO, Velusamy V, Akanmu AS. Development of trigger sensitive hyaluronic acid/palm oil-based organogel for in vitro release of HIV/AIDS microbicides using artificial neural networks. Futur. J. Pharm. Sci. 2020; 6(1): 1-14.

[35] Bhavani B, Chadalawada P, Gupta T. Formulation and Evaluation of Extended Release Mucoadhesive microspheres of atorvastatin, Indo Amer J Pharm Sci. 2015; 2(10): 1423-1436.

[36] Machado LC, daCosta D, Perotti G, Henriques R, deCouto R, Amim Jr J, Shiguihara A. Characterization of Cellulose Ether films containing Tween 20 and Tween 40. J Chem Pharm Res. 2016; 8(12):159-166.

[37] Ibekwe C, Oyatogun G, Esan T, Oluwasegun K. Synthesis and Characterisation of Chitosan /Gum Arabic nanoparticles for Bone Regeneration. Amer J Mater. Sci Eng. 2017; 5(1): 28-36.

[38] Panhwar T, Mahesar S, Kandhro A, Sheerazi S, Kori A, Laghari Z, Memon J. Physicochemical Composition and FTIR Characterization of castor seed oil. Ukr. Food J. 2019; 8(4): 778-787.

[39] Akhtar N, Khan BA, Khan MS, Mahmood T, Khan HM, Iqbal M, Bashir S. Formulation development and moisturizing effects of a topical cream of aloe vera extract. World Acad Sci Eng Technol. 2011; 5: 128-136.

[40] Ali SM, Yosipovitch G. Skin pH: From basic science to basic skin care. Acta Derm. Venereol. 2013; 93(3): 261-267.

[41] Zhang W, Yiu Y, Lin M, Luo T, Yao C. Electrical conductivity and stability of oil in water emulsions," Acta Pet. Sin. (Petroleum Process. Sect). 2008; 24(5): 592-597.

[42] Khan MF, Sheraz MA, Ahmed S, Kazi SH, Ahmad I. Emulsion separation, classification and stability assessment. J Pharm Sci. 2014; 2(2): 56-62.

[43] Olorunisola EO. Emulsions. In: Pharmaceutics in Focus - Dosage Form Development and Manufacture, 1st ed., Zaria: Ahmadu Bello University Press. 2021; 420-447.

[44] Choi SJ, McClements DJ. Nanoemulsions as delivery systems for lipophilic nutraceuticals: strategies for improving their formulation, stability, functionality and bioavailability. Food Sci Biotechnol. 2020; 29(2): 149-168. 